\title{
Increased Diameter and Enhanced Myogenic Response of Saphenous Vein Induced by Two-Week Experimental Orthostasis Are Reversible
}

\author{
G. RAFFAI ${ }^{1}$, C. LÓDI ${ }^{2}$, G. ILLYÉS ${ }^{2}$, G. NÁDASY ${ }^{1}$, E. MONOS ${ }^{1}$ \\ ${ }^{1}$ Institute of Human Physiology and Clinical Experimental Research, ${ }^{2}$ Second Department of \\ Pathology, Semmelweis University, Budapest, Hungary
}

Received December 7, 2006

Accepted January 29, 2007

On-line available February 8, 2007

\section{Summary}

Previously, increased diameter and enhanced myogenic tone were seen after 2-week 450 head-up (HUT2) in the rat. We studied the reversibility and the effect of extended tilt on this phenomenon using two experimental groups: HUT2 plus 2-week horizontal (HUT2HOR2), and 4-week tilting (HUT4). 4-weeks in normal cages (NC4) served as control. Diameter of saphenous vein (SV) in 2-20 $\mathrm{mm} \mathrm{Hg}$ pressure range, wall and media thickness, endothelial and smooth muscle cell densities, and cell proliferation were measured. The diameter of SV from HUT4 was significantly larger compared with HUT2HOR2 or NC4 within the whole pressure range both in Krebs-Ringer $(870.4 \pm 21.3$ vs. $778.2 \pm 24.9$ and $771.6 \pm 28.1 \mu \mathrm{m}$ at $10 \mathrm{~mm} \mathrm{Hg}$, respectively) and in $\mathrm{Ca}^{2+}$-free solution. Myogenic and norepinephrine-induced vascular tone, wall and media thickness did not differ among the three groups. Endothelial cell density decreased in HUT4 $(10.7 \pm 1.2)$ vs. HUT2HOR2 $(15.1 \pm 1.0)$ and NC4 $(15.3 \pm 0.6)$, while that of smooth muscle was unchanged. No cell proliferation marker was seen. In conclusion, both increased diameter and enhanced myogenic tone of SV seen in HUT2 proved to be reversible. HUT4 resulted in increased SV diameter, similarly to HUT2, however, vascular tone was not amplified. This suggests that a prolonged orthostatic load may readjust the function of smooth muscle.

\section{Key words}

Endothelial cell density • Head-up tilt • Saphenous vein • Smooth muscle cell density $\bullet$ Vascular responses

\section{Corresponding author}

G. Raffai, Institute of Human Physiology and Clinical Experimental Research, Semmelweis University, Üllói út 78/a, Budapest, Hungary H-1082. Fax.: (36-1)334-3162. E-mail: raffai@elet2.sote.hu

\section{Introduction}

Identifying the critical duration where adaptation to increased gravitational stress is still reversible might reflect the range where normal physiological vascular adaptation occurs and pathological processes start. Beyond the point of reversibility one can find responses initiating pathological alterations of the vessel wall (e.g. venous varicosity, phlebosclerosis, etc.).

Previously, we found evidence for significant functional and structural remodeling in the saphenous vein (SV) from rats tilted $45^{\circ}$ head-up in special tubecages for two weeks in order to model long-term orthostasis (for reviews see Monos et al. 1995, 2003, 2006). Different mechanisms of adaptation to the gravitational load in the SV were found which are reflected by an augmented pressure-dependent myogenic tone associated with an increased sympathetic component of smooth muscle cell membrane potential (Monos et al. 1989a, Szentiványi et al. 1997) and by an increased sympathetic innervation density (Monos et al. 2001). The enhanced tone was accompanied by increased diameter and smooth muscle cell count without significant change in wall thickness (Monos et al. 1991). A similar gravitational exposure also influenced the network properties of the saphenous vein. The diameter of main branches was increased and stable branching angles were reduced significantly at the popliteal confluence in vivo (Lóránt et al. 2003). A significant decrease in density of microvessels in the hindlimb oxidative muscles was also recognized (Hudlická et al. 1995). Recently, we found that an endothelin (ET) and platelet-derived growth factor (PDGF) containing vesicular system is selectively 
suppressed in the endothelium of rat saphenous vein by two weeks of orthostasis (Raffai et al. 2005). The enlarged SV diameter with unchanged wall thickness results in a proportional increase in elastic wall stress at identical pressure levels according to the Laplace-Frank law (Monos et al. 1989a). This chronically elevated wall stress is probably one of the key stimuli inducing local vascular adaptation (Liu et al. 2002, Lehoux 2006, Haga et al. 2007, Pries et al. 2005).

According to the reasoning above, we intended to study the reversibility of the adaptive responses seen after two weeks of head-up tiling and also the effect of an extended tilt period. Consequently, the purpose of the present investigation was

1) to compare pressure-diameter relationship of SV in rats after exposure to (a) head-up tilt for 2 weeks followed by horizontal position for additional 2 weeks, and (b) 4-week tilt with that of (c) parallel control,

2) to examine if this extended tilt enhances the myogenic tone further,

3) to analyze myogenic and adrenergic responsiveness of the vein in these groups,

4) to study changes in (a) total wall and tunica media thickness, (b) endothelial cell density and (c) smooth muscle cell density,

5 ) to test if cell proliferation is present in the vessel wall,

6) to monitor overall conditions and behavior of the animals by daily measurement of body weight, food and water consumption.

\section{Methods}

\section{Experimental animals and chronic tilt}

Adult male Sprague-Dawley rats (300-400 g body weight, $\mathrm{n}=66$ ) were kept individually either in traditional cages (normal control $=\mathrm{NC4}$ ) or in $65 \mathrm{~cm}$ long, tube like, tiltable transparent cages as described previously (Monos et al. 1989a, 2001) for 4 weeks. The animals with the tilt cages were set in $45^{\circ}$ head-up tilt (HUT) position either for 4 weeks (HUT4) or for 2 weeks followed by a confined horizontal position (HOR) for 2 weeks (HUT2HOR2). The HUT position resulted in an immediate doubling of sapheno-femoral venous pressure (Monos et al. 1989a). In both the HUT and the confined horizontal positions, the animals could freely move along their longitudinal body axis, but they were not able to turn back. They had free access to food and tap water at the top of the cage. They were removed from the cages for a 30-60 min period each day and allowed to exert their grooming behavior in traditional cages. During this period body weight, as well as daily food and water consumption were measured. At the end of the grooming period, the animals entered their cages freely, accepting it as a familiar environment. All animals were kept in a specially equipped air-conditioned room.

The investigation conforms with the Guide for the Care and Use of Laboratory animals published by the US National Institutes of Health (NIH Publication No. 85-23, revised 1996). Studies were carried out according to guidelines of Hungarian law on animal protection (243/1998), and were approved by the Semmelweis University Committee on the Ethical Use of Experimental Animals (590/99 Rh).

\section{Vessel preparation}

Saphenous veins were obtained from rats $(n=52)$ anesthetized with pentobarbital (Nembutal, $5 \mathrm{mg} / 100 \mathrm{~g}$ bw, ip). The veins from NC4 $(n=18)$, HUT2HOR2 $(n=17)$, and HUT4 $(n=17)$ were carefully dissected, and 5-7 mm long segments were removed for further investigation.

\section{Experimental setup}

The specimen was placed in a tissue bath containing normal Krebs-Ringer (KR) solution ( $\mathrm{NaCl}$ 119, $\mathrm{KCl} 4.7, \mathrm{NaH}_{2} \mathrm{PO}_{4} 0.89, \mathrm{MgSO}_{4}$ 1.17, $\mathrm{NaHCO}_{3} 24.0$, $\mathrm{CaCl}_{2} 2.5$, glucose 5.0, and EDTA $0.026 \mathrm{mmol} / \mathrm{l}$ ) bubbled with $95 \% \mathrm{O}_{2} / 5 \% \mathrm{CO}_{2}$. The vein segment was cannulated at both ends and extended to its original in situ length. The vessel was displayed on a monitor using a video camera, and its diameter was measured automatically as described elsewhere (Monos et al. 1989b, 2003, Szentiványi et al. 1997). In brief, a single video measuring-line technique was used to identify the two most pronounced contrast transitions (representing the outer vessel wall) in order to measure the distance between these transitions (being the outer vessel diameter $)$. The vessel was perfused $(0.2 \mathrm{ml} / \mathrm{min})$ and superfused $(2.5 \mathrm{ml} / \mathrm{min}$ ) by using infusion (Cole Palmer Instrument Co, Chicago, IL, USA) and peristaltic pumps (Living Systems Instrumentation, Burlington, VT, USA), respectively. Intraluminal pressure was set by changing the outflow resistance of the vessel segment.

\section{Experimental protocol}

The saphenous vein was incubated in a tissue bath at $5 \mathrm{~mm} \mathrm{Hg}$ intraluminal pressure and $37^{\circ} \mathrm{C}$ for 45 min. At the beginning of the experimental protocol, a 
norepinephrine (NE)- acetylcholine (ACh) test was performed on the preparation to prove its functional integrity. The vessel was contracted first by NE $\left(6 \times 10^{-5}\right.$ M, Sigma, St. Louis, Mo) in the superfusate, and after reaching maximal contraction it was perfused by $\mathrm{ACh}$ $\left(5.5 \times 10^{-5} \mathrm{M}\right.$, Sigma) at $5 \mathrm{~mm} \mathrm{Hg}$. The diameter of the vein segment was determined in each experimental group under control conditions (KR perfusion and superfusion) at pressure levels of 2, 5, 10, 15, and $20 \mathrm{~mm} \mathrm{Hg}$. After these pressure steps were completed, the superfusate was changed $6 \times 10^{-5} \mathrm{M} \mathrm{NE}$, finally the vessel was relaxed by perfusion and superfusion of $\mathrm{Ca}^{2+}$-free $\mathrm{KR}$ solution $(\mathrm{NaCl} 92, \mathrm{KCl}$ 4.7, $\mathrm{NaH}_{2} \mathrm{PO}_{4}$ 1.18, $\mathrm{MgSO}_{4}$ 1.17, $\mathrm{NaHCO}_{3} 24.0, \mathrm{MgCl}_{2}$ 20, glucose 5.5, EDTA 0.026, and EGTA $2.0 \mathrm{mmol} / \mathrm{l}$ ), then the pressure steps were repeated. At the end of the experiments intrinsic $\left(\mathrm{T}_{\mathrm{KR}}\right)$ and NE-induced $\left(\mathrm{T}_{\mathrm{NE}}\right)$ vascular myogenic tone was assessed by comparing the diameters with those measured in a $\mathrm{Ca}^{2+}$-free solution at each pressure level. In each case, isobar tangential strain $\left(T_{x}\right)$ was calculated as

$T_{x}=\frac{D_{x}-D_{\text {passive }}}{D_{\text {passive }}}$

where $D_{\text {passive }}$ is the external venous diameter after equilibration with $\mathrm{Ca}^{2+}$-free solution, and $\mathrm{D}_{\mathrm{x}}$ is either the diameter during normal KR perfusion and superfusion or NE superfusion at the same pressure level. Vessels exhibiting no contraction to NE, or which behaved passively in normal KR or in NE solutions were excluded from the study. In addition, vessels of maximal and minimal passive diameters were also discarded from each experimental group. After completing the experiments, the wall thickness of each vein was calculated from the diameter measured in $\mathrm{Ca}^{2+}$-free $\mathrm{KR}$ at $10 \mathrm{~mm} \mathrm{Hg}$ and from the wet weight of the excised segment using a microbalance (Cahn Instrument Inc, Cerritos, CA, USA) taking into account the specific weight of the segments $\left(1.06 \mathrm{~g} / \mathrm{cm}^{3}\right)$.

\section{Morphometric measurements}

Saphenous veins from 14 animals of the three experimental groups (NC $(n=6)$, HUT2HOR2 $(n=4)$, HUT4 $(n=4))$ were prepared for morphometric analysis after applying whole body fixation, which is required to avoid fixation artifacts (Raffai et al. 2004). The anesthetized animals (Nembutal, $5 \mathrm{mg} / 100 \mathrm{~g} \mathrm{bw}$, ip) were perfused via the aorta at $100 \mathrm{~cm} \mathrm{H}_{2} \mathrm{O}$ pressure using physiological saline, followed by $4 \%$ formaldehyde. After careful dissection, the vessels were postfixed in the same fixative solution. Saphenous vein samples were cut into three segments.

The middle segment was placed in plastic cassettes, compressed in between sheets of cardboards and layers of sponges to obtain a rectangular, flat shape. Veins in cassettes were dehydrated and embedded in paraffin. Each compressed segment was oriented perpendicularly to the cutting surface. This technique allows perpendicular sections on longitudinally oriented veins. The $4 \mu \mathrm{m}$ thick sections were stained with hematoxylin-eosin. In order to measure tunica media thickness and to identify proliferating smooth muscle cells, alpha-smooth muscle actin (dilution 1:100, \# M0851, DAKO, Glostrup, Denmark) and Ki-67 (dilution 1:50, \# M7248, DAKO) immunohistochemistry was performed using the standard streptavidin-biotinperoxidase technique with diaminobenzidine (\# K3465, DAKO), respectively. As parallel control for Ki-67 immunohistochemical specificity, the primary antibody was tested on the rat intestine. Immunostaining was found in each case in crypts of Lieberkuhn.

The two lateral cylindrical segments were cut free of most of the adventitial tissues and opened at one end. After staining with hematoxylin, these whole segments were washed in tap water, mounted with Apathy's Mounting Medium and coverslipped. For morphometric measurements digital images were captured and analyzed with Scion Image (Scion Corporation, Frederick, MD, USA) software.

\section{Determination of tunica media thickness}

Medial segment sections on slides with smooth muscle actin immunostaining were taken at final magnification of 100x. Medial areas were marked by highlighting the media using density slicing, and then their area and length were measured. Media thickness was calculated by dividing these areas with the lengths. Results were averaged.

Determination of endothelial and smooth muscle cell density

Open ends of the lateral segments of whole vein samples were snapped at final magnification of $600 \mathrm{x}$. In each case five $200 \times 200$ pixel areas $\left(0.197847 \mathrm{~mm}^{2}\right)$ were marked out, and the number of endothelial and smooth muscle cells was counted. Endothelial and smooth muscle cells were identified by their nuclear morphology. 


\section{Statistical analysis}

Results are given as means \pm S.E.M. One-way analysis of variance was used for statistical evaluation of the experimental data. Student-Newman-Keul and Dunnett's pair-wise multiple comparison procedure was used to test for significant differences between groups and within groups, respectively. $\mathrm{P}<0.05$ is considered as statistically significant.

\section{Results}

Pressure-diameter responses of HUT4, HUT2HOR2 and $\mathrm{NC4}$ in normal $\mathrm{KR}$, in NE, as well as in $\mathrm{Ca}^{2+}$-free solutions Preceding the NE-ACh test, diameter of HUT4 vessels was $859.4 \pm 25.9 \mu \mathrm{m}$ in the normal $\mathrm{KR}$ at $5 \mathrm{~mm} \mathrm{Hg}$ which was significantly higher $(\mathrm{p}<0.05)$ than that of the HUT2HOR2 $(776.1 \pm 24.9 \mu \mathrm{m})$ and the NC4 $(766.3 \pm 30.2$ $\mu \mathrm{m})$. NE significantly constricted the HUT4, HUT2HOR2, and NC4 vessels by $23.8 \pm 3.9,26.7 \pm 4.0$ and $29.7 \pm 3.9 \%$, while ACh dilated the precontracted vessels by $16.6 \pm 7.1$, $9.5 \pm 3.0$ and $11.4 \pm 2.8 \%$, respectively.

Vessel diameter of HUT4 in normal KR solution was significantly higher than that of the HUT2HOR2 and NC4 in the whole pressure range (Fig. 1a). For example at lower $(2 \mathrm{~mm} \mathrm{Hg})$ and higher $(20 \mathrm{~mm} \mathrm{Hg})$ intraluminal pressures, diameters of the veins were $836.4 \pm 21.9$ vs. $908.5 \pm 17.6 \mu \mathrm{m}$ in HUT4, $738.6 \pm 26.7$ vs. $810.5 \pm 22.0 \mu \mathrm{m}$ in HOR2HUT2, and $736.8 \pm 29.1$ vs. $820.2 \pm 23.6 \mu \mathrm{m}$ in NC4 groups, respectively.

NE caused a reduction of vessel diameter diminishing the differences between experimental groups (Fig. 1b). HUT4 remained significantly higher at $10 \mathrm{~mm} \mathrm{Hg}(760.0 \pm 34.0 \mu \mathrm{m})$ than NC4 $(632.5 \pm 36.7 \mu \mathrm{m})$ as well as at $20 \mathrm{~mm} \mathrm{Hg}(898.1 \pm 32.0 \mu \mathrm{m})$ than HUT2HOR2 $(797.7 \pm 25.9 \mu \mathrm{m})$ and $\mathrm{NC} 4(774.6 \pm 33.7 \mu \mathrm{m})$.

Similarly to normal $\mathrm{KR}$, passive diameter in $\mathrm{Ca}^{2+}$ free KR of HUT4 was significantly higher than that of the HUT2HOR2 and NC4 in the whole pressure range (Fig. 1c). For example at higher pressure $(20 \mathrm{~mm} \mathrm{Hg})$ HUT4 diameter was $938.7 \pm 19.1 \mu \mathrm{m}$, while that of the HUT2HOR2 and NC4 was $842.8 \pm 25.0$ and $851.6 \pm 21.3$ $\mu \mathrm{m}$, respectively.

Intrinsic and norepinephrine-induced myogenic tone of HUT4, HUT2HOR2 and NC4

Calculated $\mathrm{T}_{\mathrm{KR}}(8-3 \%$ from 2 to $20 \mathrm{~mm} \mathrm{Hg})$ and $\mathrm{T}_{\mathrm{NE}}$ (29-5\% from 2 to $20 \mathrm{~mm} \mathrm{Hg}$ ) did not differ substantially in the experimental and control groups (Figs 2a and 2b). a

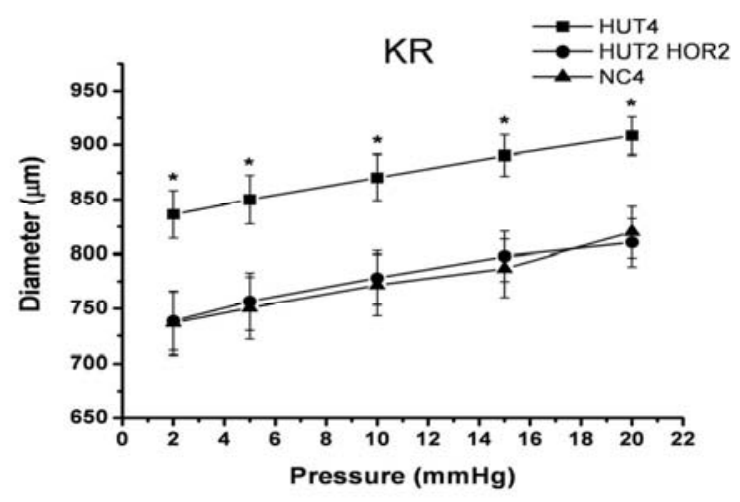

b

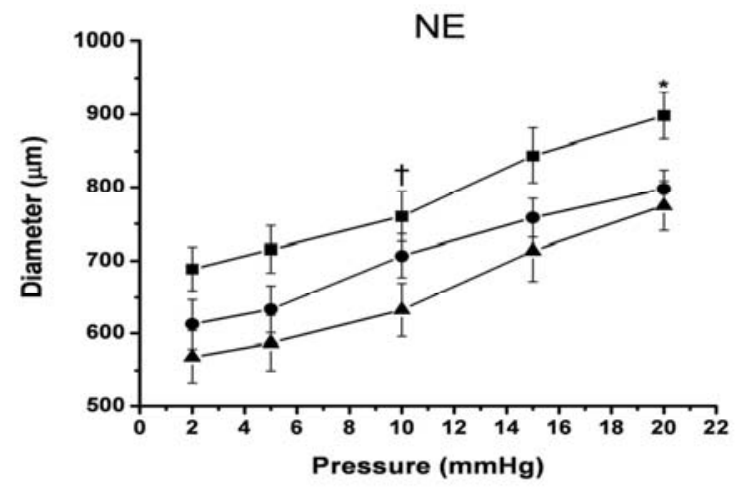

c

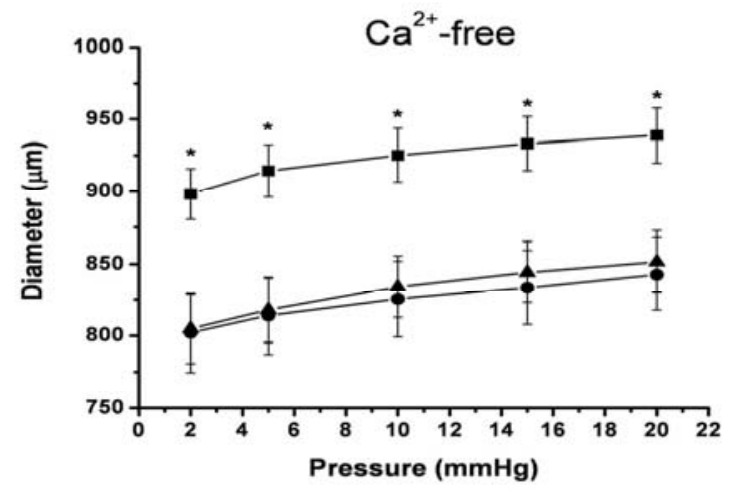

Fig. 1. External diameter of saphenous vein segments of HUT4, HUT2HOR2, and NC4 rats at 2, 5, 10, 15, and $20 \mathrm{~mm} \mathrm{Hg}$ pressure levels in (a) normal Krebs-Ringer (KR), (b) norepinephrine (NE, $\left.6 \times 10^{-5} \mathrm{M}\right)$, and (c) $\mathrm{Ca}^{2+}$-free solutions. ${ }^{*}$ or $\dagger$ shows significant differences $(p<0.05)$ from either HUT2HOR2 or NC4 alone, respectively.

\section{Thickness of the total vascular wall and the tunica media}

As shown in Figure 3a, neither the total wall nor media thickness differ between the experimental and control groups (total wall/media, NC4: 22.6 $\pm 1.5 / 6.5 \pm 0.3$, HUT2HOR2: $19.3 \pm 1 / 5.8 \pm 0.4$, HUT4: $24.7 \pm 2.1 / 6.0 \pm 0.3$ $\mu \mathrm{m})$.

\section{Endothelial and smooth muscle cell density}

Endothelial cell density was reduced to $10.7 \pm 1.2$ in HUT4 from 15.1 \pm 1.0 in HUT2HOR2 and $15.3 \pm 0.6$ in NC4. However, smooth muscle cell density showed no differences between the experimental and control groups 
a

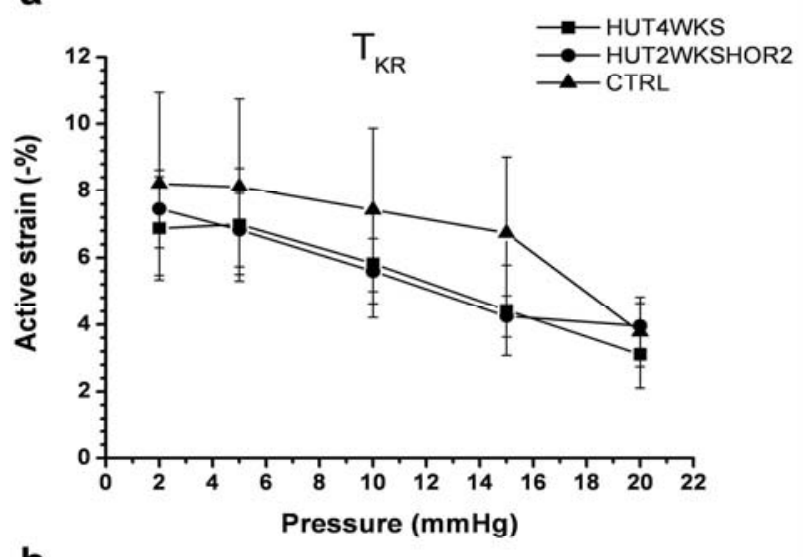

b

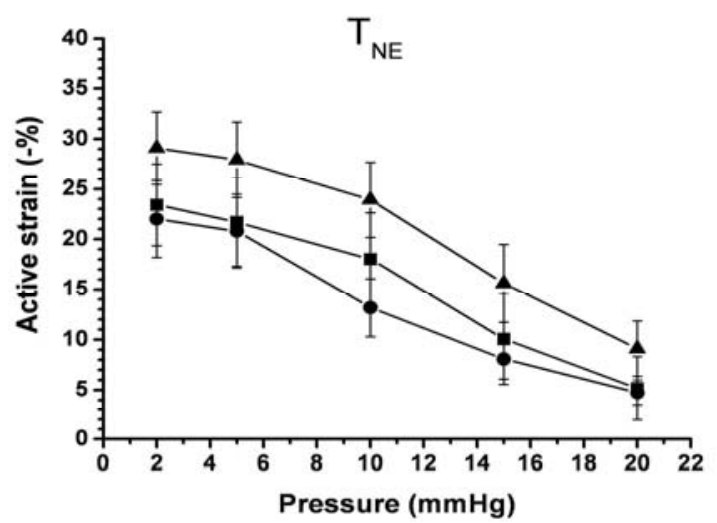

Fig. 2. Calculated (a) normal intrinsic $\left(T_{K R}\right)$ and (b) norepinephrine $\left(\mathrm{NE}, 6 \times 10^{-5} \mathrm{M}\right)$ induced $\left(\mathrm{T}_{\mathrm{NE}}\right)$ venous myogenic tone (active strain) of HUT4, HUT2HOR2, and NC4 rats at 2, 5, 10,15 , and $20 \mathrm{~mm} \mathrm{Hg}$ pressure levels.

(NC4: 20.7 \pm 1.2 , HUT2HOR2: 20.4 \pm 0.7 , HUT4: 19 \pm 3.1 , Fig. 3b).

\section{Cell kinetics: proliferation index}

No Ki-67 marker of cell proliferation was found in the endothelial and smooth muscle cells of either group (Fig. 4).

\section{Body weight, food and water consumption}

Initial body weight in the three groups was not

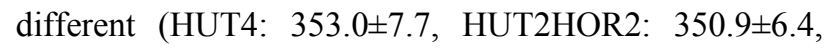
NC4: $346.7 \pm 5.3 \mathrm{~g}$ ). Tilting resulted in a small 6 and $5 \%$ reduction of body weight in HUT4 and HUT2HOR2 rats, respectively, and their value remained below that of NC4 in the course of tilting. Following this decline immediately after the onset of the tilt period, a body weight gain of similar rate as in NC4 was observed in the tilted groups, leading to significantly elevated body weight at the end of the 4-week experimental period (Fig. 5a). Similarly, a significant decline of daily chow

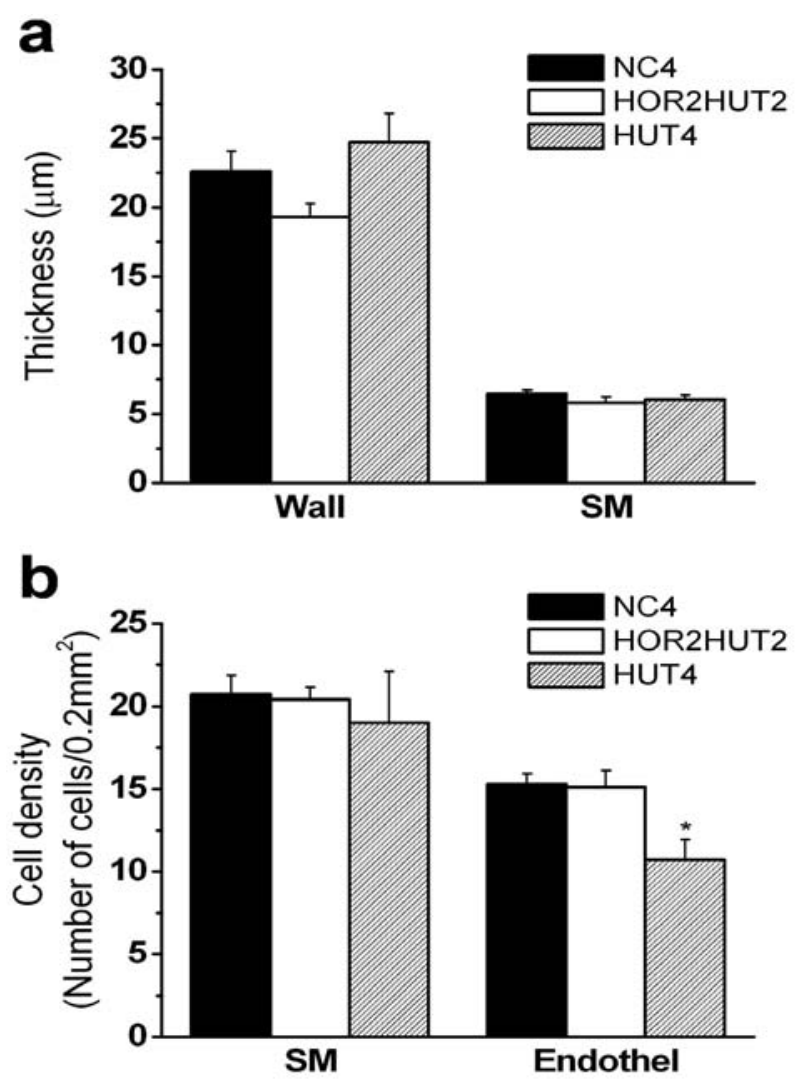

Fig. 3. Thickness of the vascular wall and the smooth muscle (SM) layer (tunica media) of saphenous veins from NC4, HUT2HOR2, and HUT4 rats (a). Smooth muscle (SM) and endothel cell densities of saphenous veins from NC4, HUT2HOR2, and HUT4 rats (b). * shows significant difference $(p<0.05)$ from HUT2HOR2 and NC4.

and $\mathrm{H}_{2} \mathrm{O}$ consumption was only observed immediately after tilting with full restoration later (Figs $5 \mathrm{~b}$ and $5 \mathrm{c}$ ).

\section{Discussion}

The most important finding of this study is that both diameter (measured in normal Krebs-Ringer and $\mathrm{Ca}^{2+}$-free Krebs-Ringer solutions) and acute pressure induced intrinsic myogenic response of saphenous vein of Sprague-Dawley rats found earlier to be increased following 2 weeks of experimental orthostasis are reversible. Diameter increase of $\mathrm{SV}$ following 2-week orthostasis (Monos et al. 1989a, 1991, Szentiványi et al. 1997, Lóránt et al. 2003) was either restored during a consecutive 2-week horizontal period (HUT2HOR2) or persisted when additional 2-week tilting (HUT4) was applied. It was reported earlier that the intrinsic myogenic response nearly doubled after 2 weeks of HUT (Monos et al. 1991, Szentiványi et al. 1997). This increased myogenic response was neither sustained in a consecutive 

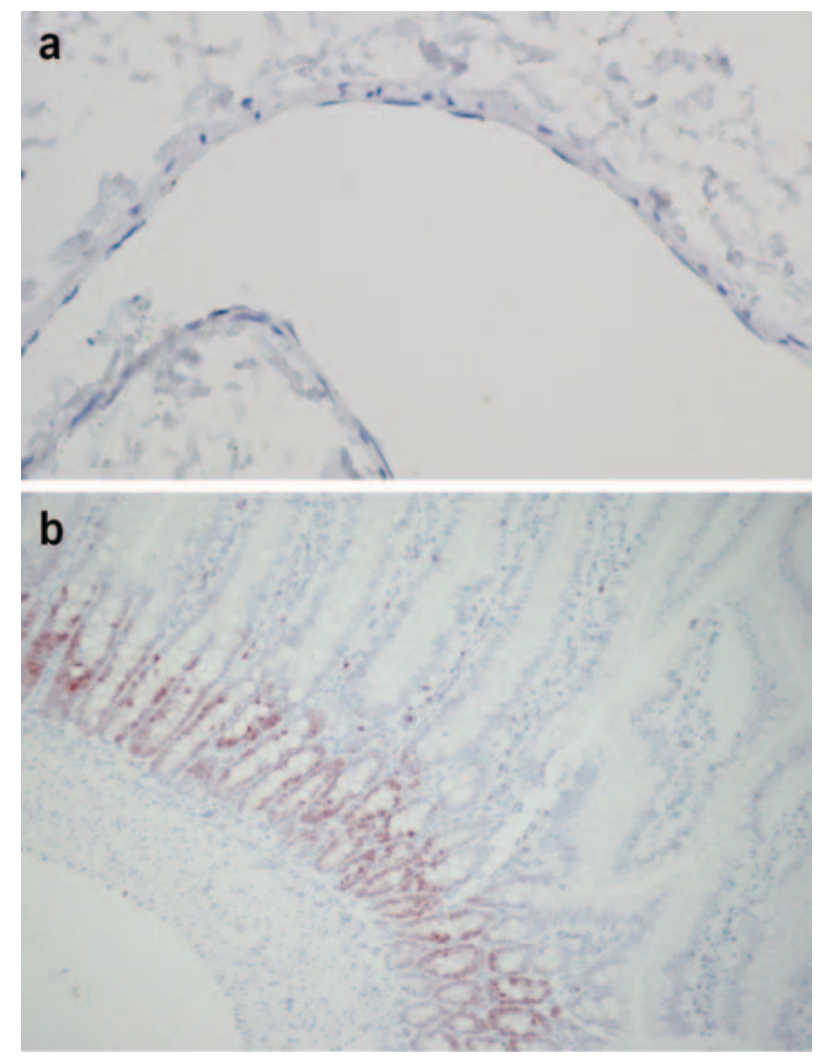

Fig. 4. Ki-67 immunohistochemical reaction is negative on the rat saphenous vein (a). Compare with the positive control slide from the intestine where proliferating cells of the crypt neck regions are evidenced in brown (b).

chronic horizontal position (HUT2HOR2) nor persisted or enhanced when tilting was prolonged by 2 weeks (HUT4), but the tone returned to the level of NC in both cases.

Similarly to the observations made in 2-week tilting experiments earlier (Monos et al. 1989a), the enlargement of the SV morphological diameter in HUT4 is probably caused by a permanently elevated hydrostatic pressure (2.9 vs. $5.9 \mathrm{~mm} \mathrm{Hg}$ ) (Monos et al. 1989a). However, this enlargement is not the consequence of a simple passive elastic expansion of the vein because its diameter was found to be increased in both $\mathrm{KR}$ and $\mathrm{Ca}^{2+}$ free KR solutions in the whole pressure range $(2-20 \mathrm{~mm}$ $\mathrm{Hg}$ ) studied. Similarly to a previous study (Monos et al. 1991), wall thickness was not altered. Moreover, as we described in this study, medial wall thickness of the extended vein (HUT4) did not differ from that of the parallel experimental (HUT2HOR2) and control groups (NC4). Consequently, during the chronic gravitational stress both adventitial and medial mass of the vein were increased approximately by $41 \%$ and $10 \%$, respectively.

These morphological and functional alterations a

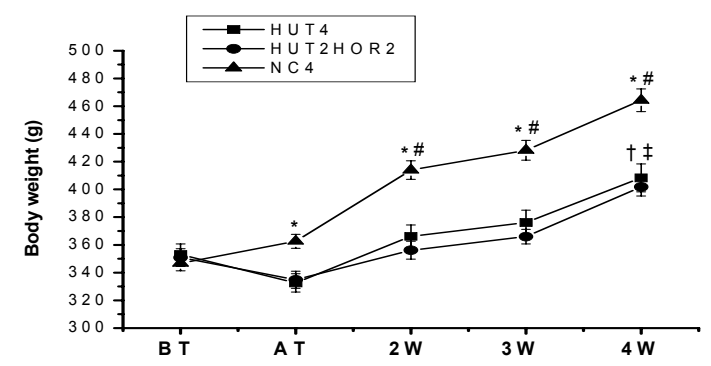

b

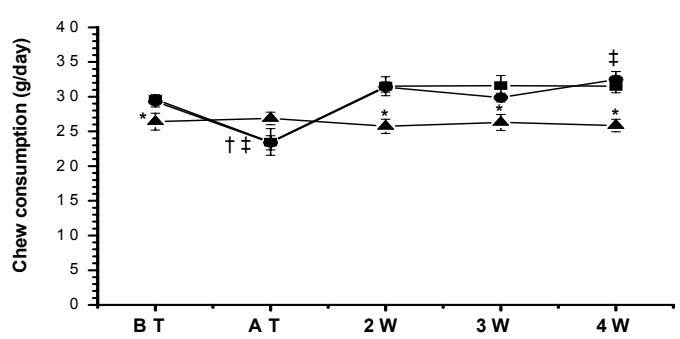

$\mathbf{C}$

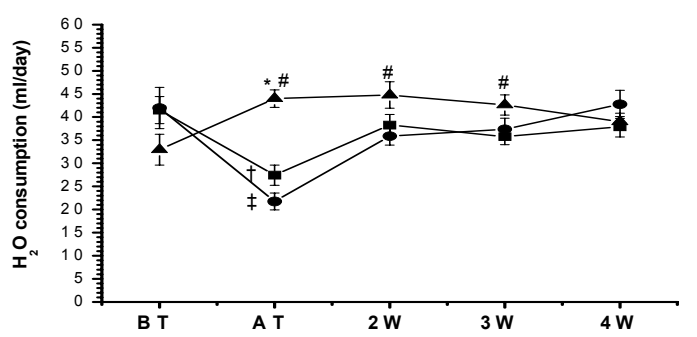

Fig. 5. Body weight (a), chow (b), and water (c) consumption of HUT4, HUT2HOR2, and NC4 rats during the experiments. BT: before tilting. AT: after start of tilting. 2W, 3W, and 4W: Week 2, 3 , and 4 of the tilt period. $* p<0.05$ different from HUT4 and HUT2HOR2. $\dagger, \ddagger$, and \# $p<0.05$ different from BT of HUT4, HUT2HOR2, and NC4, respectively.

of the SV demonstrate that similarly to pulsatile blood pressure and flow in arteries (Haga et al. 2007), a chronic rise in intraluminal pressure and in tangential wall stress may induce active adventitial and medial remodeling of the vein wall. Similar sustained hemodynamic stresses may cause structural and functional adaptation of the arterial wall (Lehoux 2006), experimental vein grafts (Monos and Csengődy 1980, Liu et al. 2002), and strangulated veins (Hayashi et al. 2003). We assume that remodeling of the venous adventitia is probably achieved by synthesis of connective tissue including collagen and elastin fibers which finally increased adventitial mass.

Because the smooth muscle density of HUT4 SV was found to be unaltered at increased vascular wall volume (that is increased diameter with unchanged wall thickness), remodeling of the media is most probably due to smooth muscle cell proliferation induced by long-term tilt. This observation is supported by findings of Liu et al. (2002) who reported significant progressive vascular cell proliferation in vein grafts following surgery. 
As we saw after 4 weeks in each experimental group, endothelial and smooth muscle cells of the SV wall did not contain Ki-67 proliferation marker, which is known to be expressed by proliferating cells in all phases of the active cell cycle $\left(G_{1}, S, G_{2}\right.$ and $M$ phase $)$ and it is absent in resting $\left(\mathrm{G}_{0}\right)$ cells (Gerdes et al. 1984). This observation suggests that instead of progressive cell proliferation (Liu et al. 2002) smooth muscle proliferation may be achieved in SV segments in the initial phase of the adaptation process. After reaching a steady-state, cell proliferation ceases and smooth muscle cells return to the resting phase $\left(\mathrm{G}_{0}\right)$ of the cell cycle which we saw at the end of the experiment. This aspect of vascular adaptation needs further investigation.

The increased SV diameter was accompanied by enlargement of the endothelial cells which was reflected by their reduced density in the tunica intima. We suppose that this dual (smooth muscle, endothelial) remodeling within the vessel wall is initiated in the endothelium which serves as a primary sensor of the hemodynamic forces in the vessel wall (Liu et al. 2002, Chien 2006). As has been hypothesized earlier, platelet-derived growth factor and endothelin synthesized by the endothelial cells are released abluminally (Raffai et al. 2005). These and other bioactive substances diffusing to smooth muscle cells exert their acute vasoconstrictor (Hughes et al. 1996, Vanhoutte 2000) and/or transient cell proliferative effects (Abedi and Zachary 1995, Hughes et al. 1996) locally.

In addition, there is evidence in the literature suggesting that vascular smooth muscle cells can also use multiple mechanisms to transduce mechanical stimuli into intracellular signals leading to modulation of gene expression and cellular function (e.g. proliferation) (Haga et al. 2007). Moreover, phenotypical modulation of endothelial and vascular smooth muscle cells that occurs in arteries during hemodynamic adaptation processes cannot be excluded (Lehoux 2006).

In the present experiments, when vessels were activated by norepinephrine, the diameter difference that existed in normal $\mathrm{KR}$ and $\mathrm{Ca}^{2+}$-free $\mathrm{KR}$ solutions between HUT4 vs. HUT2HOR2 and control (NC4) groups almost diminished in the whole pressure range studied (2-20 $\mathrm{mm} \mathrm{Hg})$. This observation suggests an enhanced adrenergic responsiveness which can be due to the increased number of smooth muscle cells following the 4-week tilting period.

Such an increased myogenic responsiveness can contribute to maintaining the diameter of $\mathrm{SV}$ against increased blood pressure/increased wall stress and thus supporting orthostatic tolerance. However, the adrenergic sensitization of vascular smooth muscle (e.g. due to increased expression of receptors) cannot be excluded. Alternatively, $\mathrm{Ca}^{2+}$-sensitization of the contractile machinery might occur and contribute to enhance adrenergic responsiveness via the RhoA/Rho-kinase pathway (Hilgers and Webb 2005).

The magnitude of isobaric active tangential strains $\left(T_{K R}\right.$ and $\left.T_{N E}\right)$ which describe relaxation of the vessels in $\mathrm{Ca}^{2+}$-free $\mathrm{KR}$ relative to normal $\mathrm{KR}$ and NE solutions remained indistinguishable among experimental groups. Tilting prolonged over 2 weeks (HUT4) could not maintain or further increase enhanced myogenic tone seen after 2 weeks (Monos et al. 1991, Szentiványi et al. 1997), instead of readjusting/normalizing it to control (NC4) levels.

Average body weight, food and water consumption showed only a negligible transient decline in the tilted groups at the beginning of the experiments, which subsequently became normalized. The body weight of NC animals was higher than in the tilted ones. This difference is probably partly due to a transient weight drop at the beginning of tilting and partly on the obviously higher physical work done by the rats kept in the tilted position.

All these changes are in accordance with our earlier observations (Monos et al. 1989a) and with those of Assenmacher et al. (1995), who demonstrated that both acute and chronic orthostatic restraint induce only a transient elevation of stress hormone levels (e.g. adrenocorticotropic hormone, corticosterone). The presence of substantial nonspecific non-gravitational chronic stress was excluded by the markedly calm behavior of the rats without showing any avoidance reactions in the tubular cages. Thus, we have good reasons to assume that morphological and functional alteration of the SV were evoked by the orthostatic body position and not by behavioral or other nonspecific effects.

In summary, we demonstrated that increases in diameter and vascular myogenic tone of rat SV following two weeks of experimental orthostasis are reversible. Furthermore, functional and morphological remodeling after prolonged (4 week) tilting resulted in an increase of adrenergic responsiveness, proliferated medial smooth muscle layer, and expanded endothelial cells, while total wall and media thickness were unaltered. Our data also show that 4 week duration of experimental orthostasis is 
not long enough to initiate severe pathological alterations in the saphenous vein.

\section{Conflict of Interest}

There is no conflict of interest.

\section{Acknowledgements}

This work was supported by Hungarian Grants: OTKA T042670/2003, ETT 128/2006, and TP-163/2005. The authors thank Ms. Ildikó Murányi for the expert technical assistance. The authors are grateful to Mr. David Byrne for proofreading and editing the manuscript.

\section{References}

ABEDI H, ZACHARY I: Signalling mechanisms in the regulation of vascular cell migration. Cardiovasc Res 30: 544$556,1995$.

ASSENMACHER I, MEKAOUCHE M, MAUREL D, BARBANEL G, GIVALOIS L, BOISSIN J, MALAVAL F, IXART G: Chronic orthostatic and antiorthostatic restraint induce neuroendocrine, immune and neurophysiologial disorders in rats. Acta Astronautica 36: 545-558, 1995.

CHIEN S: Molecular basis of rheological modulation of endothelial functions: importance of stress direction. Biorheology 43: 95-116, 2006.

GERDES J, LEMKE H, BAISCH H, WACKER HH, SCHWAB U, STEIN H: Cell cycle analysis of a cell proliferation-associated human nuclear antigen defined by the monoclonal antibody Ki-67. J Immunol 133: 1710-1715, 1984.

HAGA JH, LI YS, CHIEN S: Molecular basis of the effects of mechanical stretch on vascular smooth muscle cells. J Biomech 40: 947-960, 2007.

HAYASHI K, MORI K, MIYAZAKI H: Biomechanical response of femoral vein to chronic elevation of blood pressure in rabbits. Am J Physiol 284: H511-H518, 2003.

HILGERS RH, WEBB RC: Molecular aspects of arterial smooth muscle contraction: focus on Rho. Exp Biol Med 230: 829-835, 2005.

HUDLICKA O, DÖRNYEI G, MONOS E: The effect of long-term tilting on capillary supply in rat hindlimb muscles. Acta Physiol Hung 83: 205-212, 1995.

HUGHES AD, CLUNN GF, REFSON J, DEMOLIOU-MASON C: Platelet-derived growth factor (PDGF): Actions and mechanisms in vascular smooth muscle. Gen Pharmacol 27: 1079-1089, 1996.

LEHOUX S: Redox signalling in vascular responses to shear and stretch. Cardiovasc Res 71: 269-279, 2006.

LIU SQ, RUAN YY, TANG D, LI YC, GOLDMAN J, ZHONG L: A possible role of initial cell death due to mechanical stretch in the regulation of subsequent cell proliferation in experimental vein grafts. Biomech Model Mechanobiol 1: 17-27, 2002.

LÓRÁNT M, NÁDASY GL, RAFFAI G, MONOS E: Remodeling of the rat saphenous vein network in response to long-term gravitational load. Physiol Res 52: 525-531, 2003.

MONOS E, CSENGÖDY J: Does hemodynamic adaptation take place in the vein grafted into an artery? Pflügers Arch 384: 177-182, 1980.

MONOS E, CONTNEY SJ, COWLEY AW, STEKIEL WJ: Electrical and mechanical responses of rat saphenous vein to short-term pressure load. Am J Physiol 256: H47-H55, 1989a.

MONOS E, CONTNEY SJ, COWLEY AW, STEKIEL WJ: Effect of long-term tilt on mechanical and electrical properties of rat saphenous vein. Am J Physiol 256: H1185-H1191, 1989 b.

MONOS E, KAUSER K, CONTNEY SJ, COWLEY AW, STEKIEL WJ: Biomechanical and electrical responses of normal and hypertensive veins to short-term pressure increases. In: Cellular Aspects of Hypertension. BRUSCHI G, BORGHETTI A (eds), Springer, Heidelberg, 1991, pp 51-57.

MONOS E, BÉRCZI V, NÁDASY GL: Local control of veins: biomechanical, metabolic, and humoral aspects. Physiol Rev 75: 611-666, 1995.

MONOS E, LÓRÁNT M, FEHÉR E: Influence of long-term experimental orthostatic body position on innervation density in extremity vessels. Am J Physiol 281: H1606-H1612, 2001. 
MONOS E, LÓRÁNT M, DÖRNYEI G, BÉRCZI V, NÁDASY GL: Long-term adaptation mechanisms in extremity veins supporting orthostatic tolerance. News Physiol Sci 18: 210-214, 2003.

MONOS E, RAFFAI G, DÖRNYEI G, NÁDASY GL, FEHÉR E: Structural and functional responses of extremity veins to long-term gravitational loading or unloading - lessons from animal systems. Acta Astronautica 60: 406-414, 2006.

PRIES AR, REGLIN B, SECOMB TW: Remodeling of blood vessels: responses of diameter and wall thickness to hemodynamic and metabolic stimuli. Hypertension 46: 725-731, 2005.

RAFFAI G, FEHÉR E, NÁDASY GL, PAKU S, POGÁNY G, TÍMÁR F, SZENDE B, MONOS E: Vacuole formation in the endothelium of rat extremity vessels depends on fixation techniques and vessel type. Pathol Res Pract 200: 41-46, 2004.

RAFFAi G, FEHÉR E, NÁDASY GL, PAKU S, POGÁNY G, TÍMÁR F, SZENDE B, MONOS E: Selective suppression of an endothelin and platelet-derived growth factor containing vesicular system in endothelium of rat saphenous vein by long-term orthostasis. J Vasc Res 42: 157-164, 2005.

SZENTIVÁNYI M, BÉRCZI V, HÜTTL T, RENEMAN RS, MONOS. E: Venous myogenic tone and its regulation through $\mathrm{K}^{+}$channels depend on chronic intravascular pressure. Circ Res 81: 988-995, 1997.

VANHOUTTE PM: Say NO to ET. J Auton Nerv Syst 81: 271-277, 2000. 